\title{
¿NEOMALTHUSIANISMO O FALTA DE DESARROLLO SOCIAL? A PROPÓSITO DE POBLACIÓN Y OPORTUNIDADES EN ARICA EN LAS ÚLTIMAS DÉCADAS
}

\author{
¿NEOMALTHUSIANISM OR LACK OF SOCIAL DEVELOPMENT? ON PURPOSE \\ PEOPLE AND OPPORTUNITIES IN ARICA IN RECENT DECADES
}

\author{
Eduardo Cavieres Figueroa* y Pablo Chávez Zúñiga**
}

\begin{abstract}
Este es un trabajo preliminar sobre relaciones demográficas y socioeconómicas en la comuna de Arica, Región de Arica y Parinacota. Entendiendo los cambios en las estructuras demográficas actuales y respondiendo a preocupaciones que se basan en que el peso de la población inhibe alcanzar mejores condiciones de vida y mayor crecimiento regional, nuestro análisis considera que el problema particular de Arica, especialmente en lo que se refiere a su población juvenil, se debe mucho más a la falta de inversiones económicas y a la carencia de políticas públicas más coherentes y permanentes.
\end{abstract}

Palabras claves: Arica, estructura demográfica, población juvenil, políticas públicas.

The present preliminary work deals with the demographics and socioeconomic relationships in the commune of Arica, Region of Arica and Parinacota. Considering the changes that have affected the current demographic structures, and responding to concerns that the volume of the population hinders the access to higher living conditions and regional development, our analysis rather claims that the specific problem in Arica, especially among youth, is due to the lack of consistent and permanent public policies.

Key words: Arica, Demographic structure, youth population, public policies.

\section{Introducción:}

\section{Neomalthusianismo. Un enfoque regional}

A finales del siglo XVIII Thomas Robert Malthus publicó su conocido Ensayo sobre el principio de la población y sus efectos futuros sobre el progreso de la sociedad. El fundamento básico de su teoría consiste en que la población humana crece en progresión geométrica, mientras que los recursos para sostenerla lo hacen en progresión aritmética.

Estas ideas tuvieron tal impacto que durante el siglo XIX Darwin recogió parte de sus fundamentos para desarrollar la teoría de la "selección natural", derivando en planteamientos de carácter eugenésico y surgiendo en el siglo XX una corriente denominada neomalthusianismo. Esta perspectiva asume un nuevo foco de análisis que intenta argumentar la explotación de los trabajadores por los grupos capitalistas, las relaciones de poder entre los países desarrollados y aquellos en vías de desarrollo e, incluso, justifican las guerras imperialistas. Sus planteamientos recogen el problema malthusiano de la sobrepoblación y proponen como solución la reducción artificial de los nacimientos (anticoncepción, aborto y esterilización son los medios para lograrlo).

El ámbito económico de estos planteamientos es la escasez de recursos frente a una población que aumenta constantemente. El crecimiento demográfico conlleva la necesidad de un mayor esfuerzo en el aprovechamiento de los recursos naturales. Desde el punto de vista de la economía, se produce una revalorización del factor numérico poblacional como factor que posibilita el desarrollo. Este aumento de los habitantes produce una mayor fuerza de trabajo y es posible el mantenimiento de industrias y de las denominadas "economías de escala". Bajo esa lógica, el crecimiento económico de una comunidad se mantiene al conservar una población activa más numerosa que aquella no activa (niños y ancianos).

En la actualidad, el problema no consiste en una superpoblación que pueda terminar con los recursos alimenticios, sino más bien en los daños que podrían llegar a provocar la aplicación de una óptica antinatalista en una región. Sin duda, los

\footnotetext{
* Investigador Invitado de la Universidad de Tarapacá. Correo electrónico: ecaviere@ ucv.cl

** Programa de Doctorado en Historia de la Universidad de Chile. Correo electrónico: pablo.chavez.zuniga@gmail.com
} 
índices de natalidad se encuentran en un descenso continuo, sobre todo en aquellas regiones que se encuentran en una transición demográfica avanzada y en una disminución de la natalidad que no permite el recambio generacional. En ese caso, los propósitos se están enfocando en "educar" a la opinión pública en el sentido que el crecimiento de la población es una amenaza para mantener el equilibrio medioambiental. Siguiendo los planteamientos de Sánchez (2008), se la quiere convencer de que el exceso de población y su voracidad consumista es la razón del deterioro ecológico. Pretenden que asumamos que el término población es sinónimo de contaminación y de destrucción de especies. Esta óptica profundiza el análisis y genera una discusión sobre los modelos construidos por los medios de comunicación, ya que la defensa del medio ambiente se utiliza como argumento para justificar los programas de control demográfico.

Ahora bien, el enfoque espacial que asumen los trabajos desde el neomalthusianismo aborda extensas áreas de terreno vinculadas a países y continentes. Esta investigación propone un área comunal periférica bastante acotada y el desarrollo de las variables demográficas en Arica, como región particular. De esa manera, el enfoque estará en la aplicación de las políticas sociales y sus consecuencias en la población, por sobre las temáticas relacionadas a lo medioambiental.

Las estadísticas de la población conforman el pronóstico de un aspecto fundamental en torno al que se desarrollan las actividades económicas y sociales de una región. Desde el punto de vista de las políticas públicas, la cantidad de recursos que se invierten en infraestructura económica y social se encuentran relacionados con las proyecciones demográficas. Sin lugar a dudas, estas entregan información fundamental para planificar gastos y decidir sobre las políticas públicas. La Región de Arica y Parinacota fue creada el 2007 por la Ley $\mathrm{N}^{\circ} 20.175$, lo que produjo una reorganización político-administrativa de la antigua Región de Tarapacá. De esa manera, la naciente región quedó constituida por dos provincias (Arica y Parinacota) y 4 comunas (Arica, Camarones, Putre, General Lagos). El análisis que se presenta a continuación está centrado solo en la comuna de Arica.

Durante los últimos años, la ciudad de Arica se ha transformado es un espacio de estudio sobre las dinámicas de población, lo que ha provocado la proliferación de libros y publicaciones de diferentes especialidades que abordan esta temática. Entre las investigaciones que han puesto atención a los procesos poblacionales se encuentran los trabajos de Ruz, Díaz y Galdames (Ruz et al., 2012; 2008) y Marcela Tapia Ladino. La propuesta metodológica de ambas investigaciones corresponde a la utilización de los datos censales y la revisión bibliográfica especializada en la temática aplicada a los espacios fronterizos.

\section{La estructura de población de Arica en las últimas décadas}

En los Gráficos presentados a continuación aparece la composición de la población distribuida por segmentos de edad (eje vertical de ordenadas) y sexo (eje horizontal de abscisas). A partir de este histograma doble se pueden deducir varias otras informaciones demográficas, como migraciones, mortalidad infantil, conjeturas sobre sectores entrantes, políticas vinculadas a la natalidad, etcétera.

Hacia 1990 la comuna de Arica tenía 167.582 habitantes, el 50,06\% masculino y el $49,93 \%$ femenino. El segmento poblacional infantojuvenil (menores de 15 años) alcanzaba 50.606 habitantes. La sección de la población considerada como fuerza de trabajo (entre 15 y 64 años) tenía 109.689 habitantes (ver Gráfico 1). La población adulta mayor (más de 65 años) estaba conformada por 7.287 habitantes.

En el sector de la barra del segmento 10-14 años, nacidos entre 1976 y 1980, se puede observar un entrante en la pirámide, mientras se aprecia un aumento importante en la población masculina de entre 15-19 años, produciéndose desde allí una disminución constante de cada tramo de edad hasta la parte final de la pirámide.

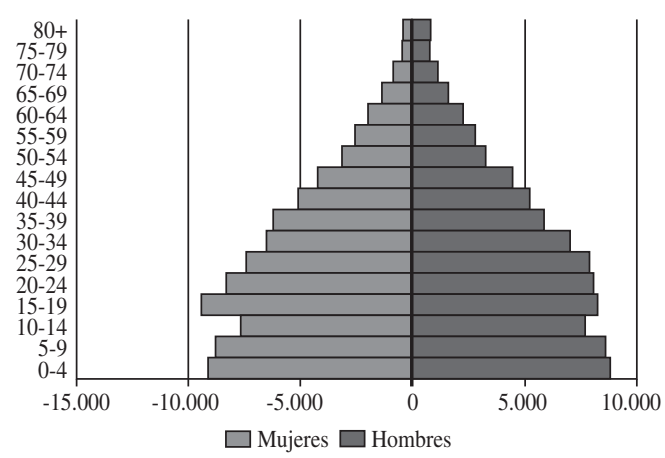

Gráfico 1. Pirámide población por sexo, comuna de Arica. Según grupos quinquenales de edad, 1990.

Fuente: elaboración propia a partir de las cifras entregadas por el Instituto Nacional de Estadísticas (INE). 
Esta pirámide corresponde a una forma de pagoda, manifiesta una destacada importancia de la población joven, tiene una base ancha que nos indica una alta natalidad, y una disminución a medida que nos acercamos a la cúspide o punta estrecha, es decir, escasa población anciana, manifestación de una tasa de mortalidad elevada y una baja esperanza de vida, se trata de un modelo de sociedad subdesarrollada.

En la base de la pirámide se destaca un número mayor de población masculina respecto de la femenina. Al calcular la tasa de masculinidad para este segmento podemos señalar que la cantidad resultante es de 104,2 varones existentes por cada 100 mujeres. Esta relación se encuentra equilibrada en los sectores medios de la pirámide, pero se va alterando a favor de las mujeres a medida que ascendemos hacia la cúspide. Si esta fuera una comunidad económicamente desarrollada, el número de mujeres mayores de 85 años duplicaría a los hombres, pero, en este caso, se produce una diferencia con una población femenina de 741 habitantes y una masculina de 441 habitantes, por lo tanto reafirmamos un modelo de sociedad subdesarrollada.

¿Qué sucedía al llegar a la década siguiente? En el 2000 la comuna tenía 189.005 habitantes, de estos el 49,7\% pertenecía al sexo masculino y el 50,29\% al sexo femenino (ver Gráfico 2). El segmento poblacional infantojuvenil alcanzaba a 52.937 habitantes. La sección de la población considerada como fuerza de trabajo (entre 15 y 64 años) tenía 124.218 habitantes. La población adulta mayor (más de 65 años) estaba conformada por 11.850 habitantes.

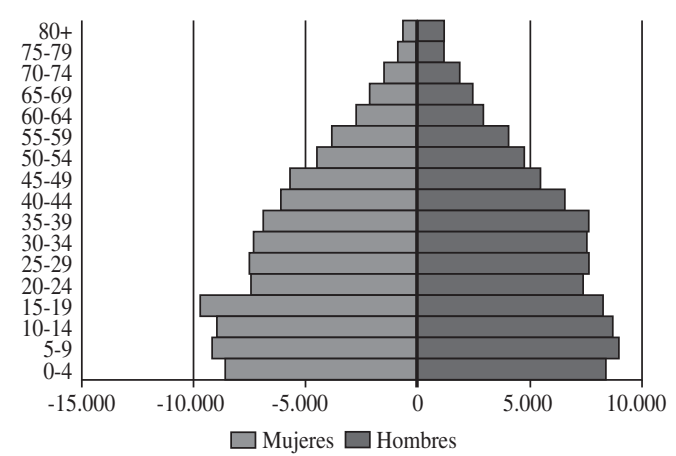

Gráfico 2. Pirámide población por sexo, comuna de Arica. Según grupos quinquenales de edad, 2000.

Fuente: elaboración propia a partir de las cifras entregadas por el Instituto Nacional de Estadísticas (INE).
Al comparar la figura de esta pirámide con las anteriores podemos seguir distinguiendo una de tipo progresiva en forma de pagoda, aunque su base es menor y la cúspide es mayor a la pirámide de 1990. En el caso particular de esta representación no hay ningún segmento que tenga una entrada específica, aun cuando para los nacidos entre 1981 y 1985 existe una cantidad bastante mayor de hombres respecto de las mujeres. La tasa de masculinidad registra 117,1 hombres por cada 100 mujeres.

En el 2010 la comuna mostraba su descenso poblacional llegando a 180.754 habitantes: $48,2 \%$ masculino y 51,79\% femenino (ver Gráfico 3). El segmento poblacional infantojuvenil alcanzaba a 42.378 habitantes. La sección de la población considerada como fuerza de trabajo (entre 15 y 64 años) tenía 120.289 habitantes. La población adulta mayor (más de 65 años) estaba conformada por 18.087 habitantes.

En este caso la pirámide corresponde ya a un modelo de población estancada y muestra una forma de campana. Se trata de una población mayoritariamente adulta. Apreciamos una base menos ancha porque la tasa de natalidad ha ido disminuyendo, entre el segmento de inicio en los 0 años y el grupo que finaliza en los 14 años es posible distinguir esta base más delgada. El tramo que va de los 15-19 años es el sector más grueso de la muestra y de ahí en adelante disminuye de manera constante en dirección a la parte superior de la pirámide por el aumento en la esperanza de vida y el descenso de las tasas de mortalidad.

La población masculina es mayor a la femenina hasta la sección que va entre 25 y 29 años, en el

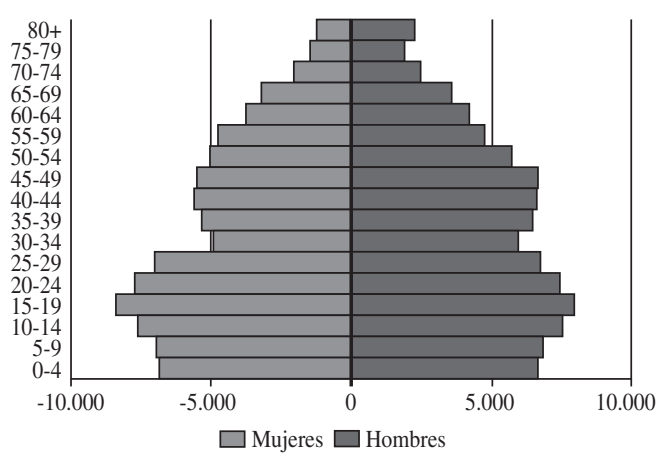

Gráfico 3. Pirámide Población por sexo, comuna de Arica. Según grupos quinquenales de edad, 2010.

Fuente: elaboración propia a partir de las cifras entregadas por el Instituto Nacional de Estadísticas (INE). 
tramo siguiente (30-34 años) la población masculina disminuye ostensiblemente, desde ahí en adelante la cantidad de mujeres siempre supera a la de hombres de manera clara. Por ejemplo, en la parte final de esta pirámide, en la población mayor de 80 años, al calcular la tasa de masculinidad obtenemos que por cada 54,5 hombres existen 100 mujeres.

Según las proyecciones del INE, en el 2015 la población de la comuna de Arica seguirá disminuyendo, llegando a 171.918 habitantes, de ellos solo el 47,15\% pertenecerá al sexo masculino y el 52,84\% al sexo femenino (ver Gráfico 4). El segmento poblacional infantojuvenil alcanzaría a los 37.816 habitantes. La sección de la población considerada como fuerza de trabajo (entre 15-64 años) tendría 112.870 habitantes. La población adulta mayor (más de 65 años) estaría conformada por 21.232 habitantes.

Siguiendo las proyecciones proporcionadas por el INE en este modelo, una vez más se presenta una pirámide estancada en forma de campana. A simple vista hay dos elementos que resultan destacables: el primero, el crecimiento del sector superior de la pirámide, es decir, la población mayor de 65 años. La segunda característica es la superioridad de la población femenina por sobre la masculina a partir del segmento 25 a 29 años.

Para demostrar el envejecimiento de la comunidad conviene calcular la tasa de dependencia de la población envejecida, para establecer la relación entre los sectores dependientes y los sectores productivos, de la que aquella depende. Esta tasa se obtiene al dividir el número de personas mayores de 65 años por el total de la población que se encuentra entre 15 a 64 años, este resultado se multiplica por cien. Para el caso de Arica en el 2015 la tasa de dependencia de la población envejecida señala que por cada 100 habitantes en edad de trabajar (15 a 64 años) existirán 18,8\% de adultos mayores de 65 años.

En consecuencia, en 25 años, Arica muestra un cambio notable en términos de su estructura básica de población:

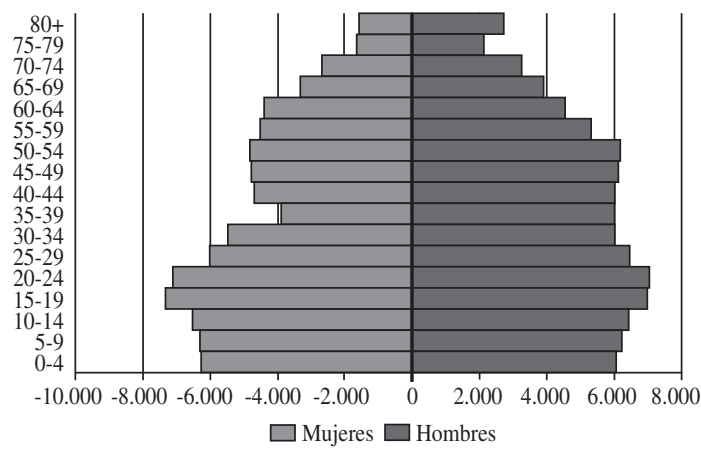

Gráfico 4. Pirámide población por sexo, comuna de Arica. Según grupos quinquenales de edad, 2015.

Fuente: elaboración propia a partir de las cifras entregadas por el Instituto Nacional de Estadísticas (INE).

¿Se trata solo de cambios demográficos producto de modificaciones puramente demográficas? Sabemos de los nuevos comportamientos respecto de los índices básicos de natalidad y mortalidad, pero en el caso de Arica el muy lento crecimiento de la población ubicada entre los 15 y los 64 años de edad, el aumento de los mayores de 65 años y la superioridad del índice de feminidad sobre el de masculinidad obedecen muy marcadamente a cuestiones relacionadas con el estancamiento del crecimiento económico de la ciudad (ver Tabla 1).

\section{Estadísticas Económico-Sociales de la Comuna de Arica}

Los índices de pobreza y de pobreza extrema, indigencia, superan las cifras medias nacionales, presentando 18,6\% de pobreza total. Ya en el 2006 la Encuesta CASEN observaba una diferencia de 12,2 puntos entre la pobreza rural y la urbana, una de las mayores distancias a nivel nacional. Según el mismo instrumento, a fines del 2011 la población que se encontraba en situación de pobreza en la Región de Arica y Parinacota alcanzaba a 27.581

Tabla 1. Trayectoria población por segmentos de edad, comuna de Arica 1990-2015

\begin{tabular}{cccccc}
\hline Años & Población total & Población -15 años & Población 15-64 años & Población + 65 años & $\begin{array}{c}\text { Relación población } \\
\text { masculina/femenina (\%) }\end{array}$ \\
\hline 1990 & 167.582 & 50.606 & 109.689 & 7.287 & $50.06 / 49.94$ \\
2000 & 189.005 & 52.937 & 124.218 & 11.850 & $49.70 / 50.30$ \\
2010 & 180.754 & 42.378 & 120.289 & 18.087 & $48.20 / 51.80$ \\
2015 & 171.918 & 37.816 & 112.870 & 21.232 & $47.15 / 52.85$ \\
\hline
\end{tabular}


personas, $15,7 \%$ de la población regional ${ }^{1}$. En este segmento, la población que se encontraba en situación de pobreza extrema llegaba a 3.762 personas, el 2,1\% de la población regional. Por cierto, había una correlación respecto de los más bajos indicadores de ocupación e nivel nacional: $8,6 \%$ de desocupación en el último trimestre de $2008^{2}$, una misma correlación en términos de la tasa de participación laboral: $53,1 \%$ para la región; $55,9 \%$ para nivel país (ver Gráfico 5).

Una buena síntesis de las diferencias entre la región y el centro del país (Región Metropolitana) se puede observar por la comparación de los ingresos y el coeficiente de Gini ${ }^{3}$. En la primera columna de la siguiente Tabla aparecen los ingresos per cápita en 1990, cuando tanto la Región Metropolitana como la entonces Tarapacá superaban al promedio nacional. En el 2003 los ingresos en el centro del país aumentaron en 5,8\% y a nivel nacional $4,2 \%$; en cambio, a nivel de la región estudiada aquel aumento no alcanzó al 1\%. Aún así, en ese mismo año, al contrastar la información de los ingresos con el coeficiente de Gini se observa que la distribución de ingreso en la región de Tarapacá tendía a una mayor igualdad mostrando índices más cercanos a cero que la Región Metropolitana y el promedio nacional: ¿pobreza generalizada?

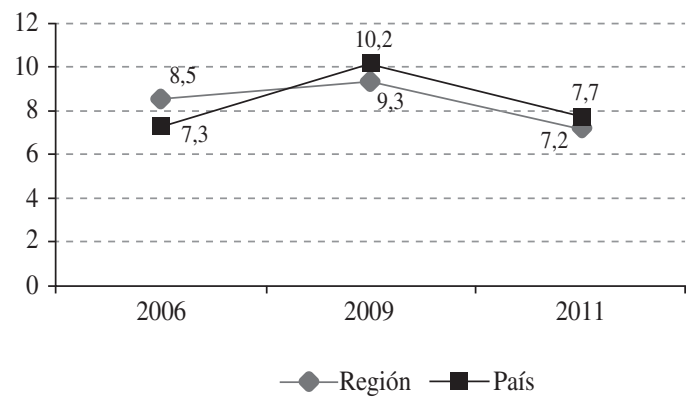

Gráfico 5. Tasa de desocupación, 2006-2011.

Fuente: Ministerio de Desarrollo Social, Resultados Regionales Encuesta Casen 2011: Región de Arica y Parinacota, p. 8.
Como se ha señalado en las estadísticas anteriores, el número de la población laboral fue creciendo muy lentamente en las últimas dos décadas y, en ella, según la Encuesta Casen, en el 2011, la población joven (18 a 29 años) representaba el 20,0\% de las personas residentes en viviendas particulares en la Región de Arica y Parinacota, proporción similar a la registrada a nivel nacional $(20,5 \%)$. No obstante, respecto de la evolución de la tasa de ocupación por tramo etario se aumentaba la persistente brecha existente entre los jóvenes de 18 a 29 años de edad con las de 30 y más años: en ese año, la tasa de ocupación de los jóvenes alcanzó al 41,8\%, mientras que la de las personas de 30 años y más llegó a $57,9 \%$ (ver Gráfico 6).

En realidad, las cifras básicas de población y sus tendencias no deberían causarnos asombros. En lo general, es lo que sucede. Lo que sí requiere de mayor preocupación es lo concerniente a las proyecciones de esta estructura de población, y particularmente a lo que va sucediendo con el segmento joven de la misma. Es usual que estando en Arica mucha gente adulta siente que está sola porque sus hijos, especialmente

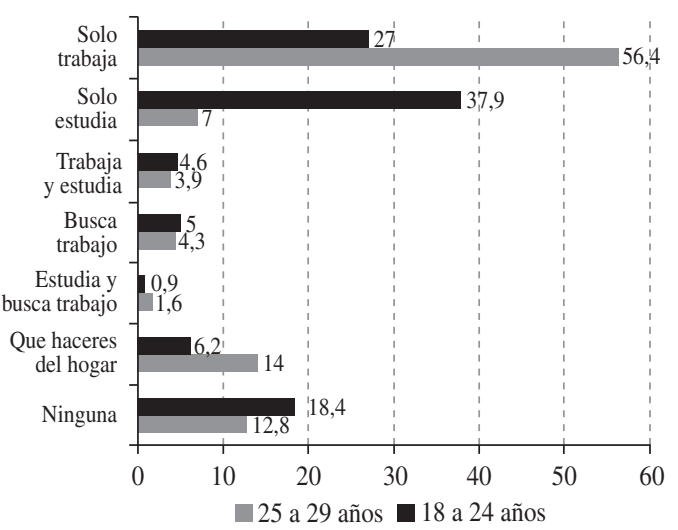

Gráfico 6. Distribución de actividades de los jóvenes de 18 a 29 años, por grupo de edad, 2011 (en porcentajes).

Fuente: Ministerio de Desarrollo Social, Encuesta Casen.

Tabla 2. Comparación ingresos y coeficiente de Gini entre Región Metropolitana y Tarapacá (Arica y Parinacota)

\begin{tabular}{lcccccrr}
\hline \multicolumn{1}{c}{ Región } & Ingresos & Ingresos & Ingresos & Dif. & Gini & Gini & Población \\
& 1990 & 2003 & 2003 (USD) & & 1990 & 2003 & \\
\hline Metropolitana & 110.129 & 193.199 & 403 & $5,80 \%$ & 0,57 & 0,58 & 6.685 .685 \\
Tarapacá (Arica y Parinacota) & 116.127 & 129.249 & 269 & $0,87 \%$ & 0,55 & 0,50 & 513.837 \\
Nacional & 92.391 & 143.705 & 299 & $4,27 \%$ & 0,58 & 0,57 & 16.634 .603 \\
\hline
\end{tabular}


quienes han logrado un nivel de escolaridad superior a la media, emigra naturalmente y trata de buscar nuevos horizontes fuera de la ciudad.

¿Se trata de un problema solo de carácter educacional? ¿Poco acceso a la educación? Las estadísticas desmienten aquello. Durante la década de los años noventa el Estado inició una serie de reformas en el ámbito educativo cuya finalidad era lograr el acceso universal a la educación básica y al mejoramiento de su calidad y equidad. En los últimos años ha disminuido el número de analfabetos, han aumentado los años de escolarización y la cobertura educativa en la población; sin embargo, en la actualidad persisten apreciables desigualdades educacionales (ver Gráfico 7).

En este nivel la región presenta indicadores mejores que la media del país con una tasa de alfabetismo de $97,6 \%$, años de escolaridad 10,5, y cobertura educacional de $78,1 \%{ }^{4}$. La tasa de

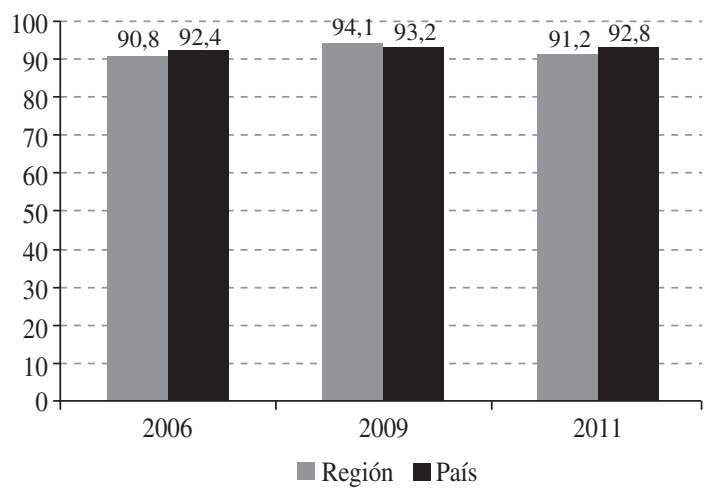

Gráfico 7. Cobertura educacional neta en educación básica, 20062011. Fuente: Ministerio de Desarrollo Social, Resultados Regionales Encuesta Casen 2011: Región de Arica y Parinacota, p. 12.

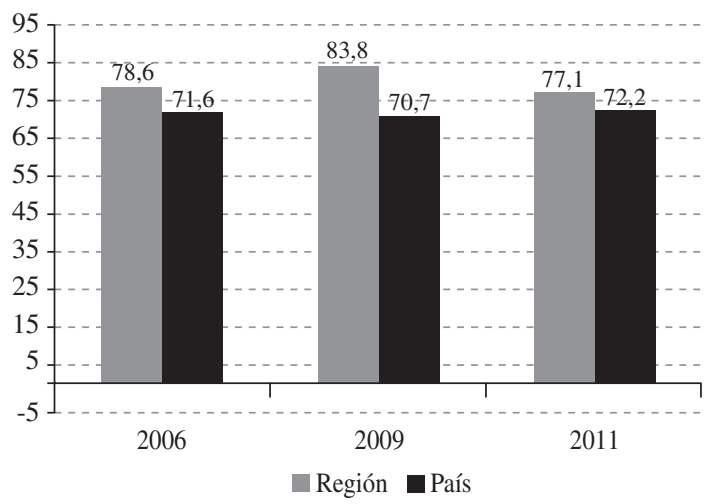

Gráfico 8. Cobertura educacional neta en educación media, 2006-2011. Fuente: Ministerio de Desarrollo Social, Resultados Regionales Encuesta Casen 2011: Región de Arica y Parinacota, p. 13. analfabetismo ha disminuido en los últimos cinco años, alcanzando un nivel inferior (alrededor de $1 \%$ ) al promedio en el país. Por su parte, la cobertura neta de la educación básica en la región se ha mantenido sobre el $90 \%$ de la población desde el 2006 y en niveles similares a los que se observan a nivel nacional (ver Gráfico 9).

En términos de cobertura neta de la educación media en la región, esta presenta cifras positivas entre 2006 y 2011, alcanzando en el último año a 77,1\%, índice igualmente superior a la registrada en el país $(72,2 \%)$. No hablamos acá de los niveles de deserciones del sistema que, igualmente en este último año, llegó a 39,7\% de los ingresados. Durante todo el período analizado, el nivel de este indicador regional fue más bajo que la media del país (ver Gráfico 8).

Finalmente, mientras la cobertura neta de educación superior a nivel nacional registró un incremento sostenido, de más de 5 puntos porcentuales entre 2006 y 2011, en la Región de Arica y Parinacota esta cobertura descendió en más de un punto. Un análisis de los registros de ingreso a la Universidad de Tarapacá pueden mostrar interesantes puntos de vista para pensar en esta situación. No se trata de problemas de ingreso, sino de pertenencia y residencia de los jóvenes en las comunas de la región.

Por lo tanto, ¿problema de envejecimiento o problema con las expectativas juveniles? (ver Gráfico 10) El aumento de la población adulto mayor es un hecho excepcional en la Historia de Chile, considerando que a inicios del siglo XX la esperanza de vida era de 35 años, aproximadamente. El segmento de población adulto mayor aumenta a partir de la segunda mitad del siglo XX producto de los cambios económicos, sociales y culturales acaecidos en el

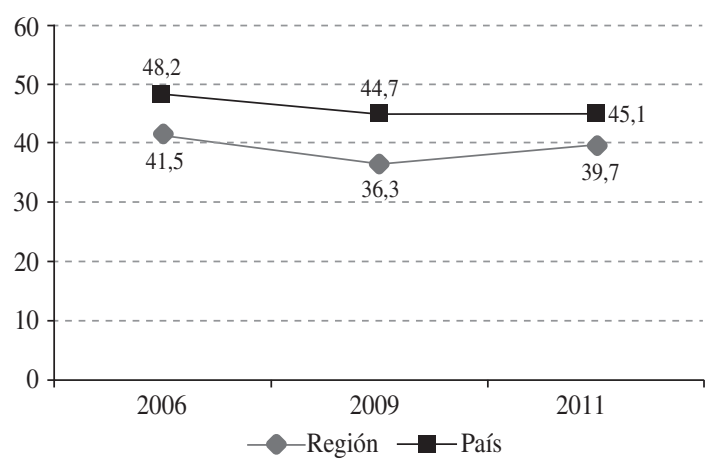

Gráfico 9. Porcentaje de la población con nivel educacional media incompleta o inferior, 2006-2011.

Fuente: Ministerio de Desarrollo Social, Resultados Regionales Encuesta Casen 2011: Región de Arica y Parinacota, p. 11. 


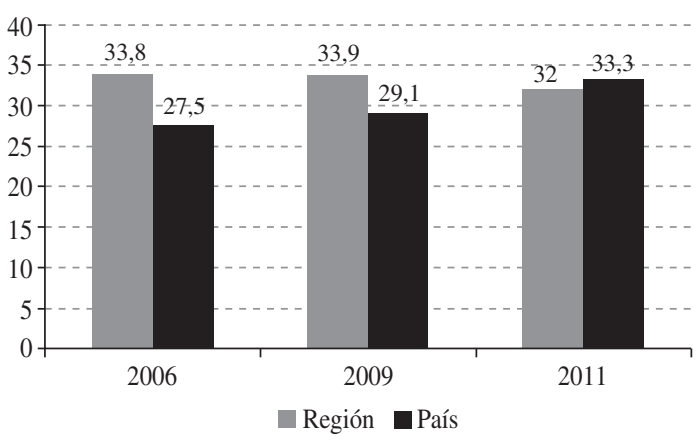

Gráfico 10. Cobertura educacional neta en educación superior, 2006-2011.

Fuente: Ministerio de Desarrollo Social, Resultados Regionales Encuesta Casen 2011: Región de Arica y Parinacota, p. 13.

país, realidad que incluye a la ciudad de Arica. De ese modo se produce una modificación en el perfil demográfico del país, lo que posiciona a Chile en una etapa de transición demográfica tendiente al envejecimiento de la población caracterizada por una natalidad y mortalidad relativamente bajas y una mayor esperanza de vida al nacer.

Las diferencias entre lo nacional y lo regional se basan en el movimiento de la población, particularmente del sector juvenil de la misma. Entre las regiones con migración positiva en 2002 la región de Tarapacá alcanzó 0,6 por mil y ocupó el lugar sexto. Esta región en los últimos censos de población ha ido experimentando una tendencia que se detiene respecto de la salida de porcentajes importantes de sus jóvenes, pero en términos de números la salida de quienes han alcanzado un grado profesional sigue siendo importante (ver Gráficos 11 y 12).

¿Cuáles son las principales regiones de destino a nivel nacional del segmento masculino migrante? Según el Censo 1992, la región de Tarapacá en el período 1987-1992 entregó el 36\% (6.119 habitantes) de sus emigrantes hombres a la Región Metropolitana, siendo la zona que concentra el mayor índice de movilidad durante este período. Seguida por el $18,5 \%$ (3.150 habitantes) de sus emigrantes hombres a la región de Antofagasta y el 14,8\% (2.513 habitantes) a la región de Valparaíso. De esa manera, en este quinquenio 16.985 habitantes que se encontraban en 1987 en la región de Tarapacá se desplazaron, principalmente, hacia estas tres regiones (ver Tabla 3 ).

En cuanto al Censo 2002, la región de Tarapacá en el período 1997-2002 tuvo el 31,7\% (7.069 habitantes) de sus emigrantes masculinos a la Región Metropolitana. En forma similar a lo acontecido en 1992, en 2002 esta región ha constituido un foco

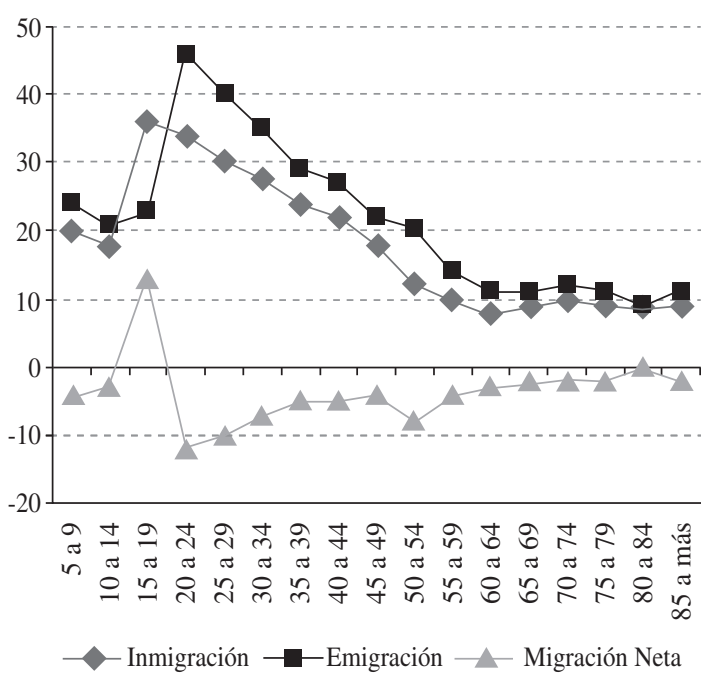

Gráfio 11. Tasas de migración hombres. Región de Arica y Parinacota, período 1997-2002 (tasa por mil personas).

Fuente: Instituto Nacional de Estadísticas (INE), Población y migración interna. Características sociodemográficas 1997-2002, p. 77.

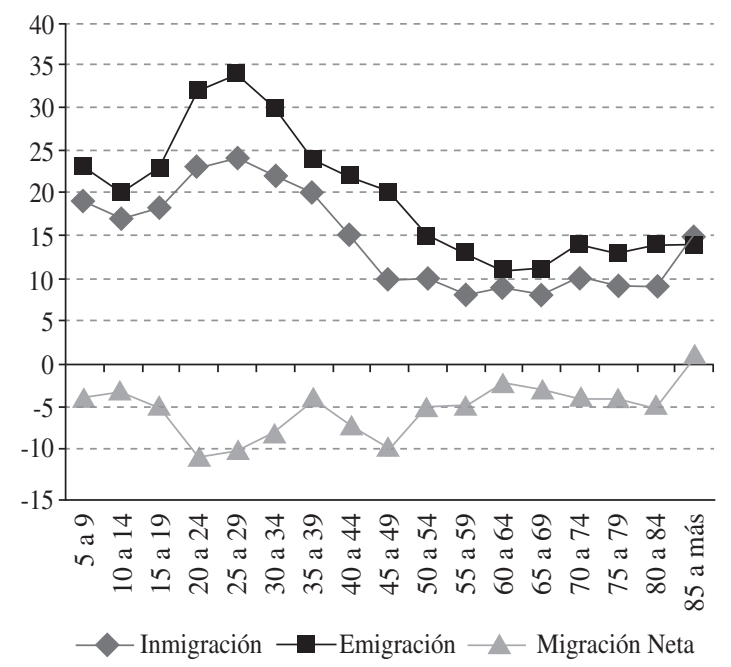

Gráfico 12. Tasas de migración mujeres. Región de Arica y Parinacota, período 1997-2002 (tasa por mil personas).

Fuente: Instituto Nacional de Estadísticas (INE), Población y migración interna. Características sociodemográficas 1997-2002, p. 78.

para la región del extremo norte. Las siguientes mayorías son la región de Antofagasta y Valparaíso, con $18,5 \%$ y $15,1 \%$, respectivamente. Para este quinquenio se produjo un desplazamiento interno desde Tarapacá hacia otras regiones del país correspondiente a 22.274 habitantes (ver Tabla 4).

En el caso femenino, siguiendo el Censo 1992, la región de Tarapacá entregó de sus emigrantes mujeres el 36,3\% (5.442 habitantes) a la Región Metropolitana, 
Tabla 3. Población masculina de 5 años o más: corrientes migratorias observadas, región Tarapacá. 1987-1992. Censo 1992

\begin{tabular}{lc}
\hline $\begin{array}{l}\text { Región residencia habitual } \\
1992\end{array}$ & $\begin{array}{c}\text { Región residencia habitual } \\
\text { en } 1987 \text { (Tarapacá) }\end{array}$ \\
\hline Antofagasta & $18,5 \%(3.150$ hab.) \\
Valparaíso & $14,8 \%(2.513$ hab.) \\
Metropolitana & $36,0 \%(6.119$ hab.) \\
Población total emigrante & 16.985 habitantes \\
\hline
\end{tabular}

Fuente: Instituto Nacional de Estadísticas, Migraciones Internas Regionales 1992-2002.

Tabla 4. Población masculina de 5 años o más: corrientes migratorias observadas, región Tarapacá. 1997-2002. Censo 2002

\begin{tabular}{lc}
\hline $\begin{array}{l}\text { Región residencia habitual } \\
2002\end{array}$ & $\begin{array}{c}\text { Región residencia habitual } \\
\text { en } 1997 \text { (Tarapacá) }\end{array}$ \\
\hline Antofagasta & $18,5 \%(4.110$ hab.) \\
Valparaíso & $15,1 \%(3.370$ hab.) \\
Metropolitana & $31,7 \%(7.069$ hab.) \\
Población total emigrante & 22.274 habitantes \\
\hline
\end{tabular}

Fuente: Instituto Nacional de Estadísticas, Migraciones Internas Regionales 1992-2002.

el 18,5\% (2.780 habitantes) a la de Antofagasta y el 14,5\% (2.175 habitantes) a la región de Valparaíso. Así, entre los años 1987 y 1992 el total de la población femenina migrante es de 15.011 habitantes (ver Tabla 5).

Para el Censo 2002 la población femenina migrante corresponde al 31,3\% (5.709 habitantes); a la Región Metropolitana, el 17,9\% (3.268 habitantes), el 16,4\% a la región de Valparaíso y el 12,0\% (2.200 habitantes) a la región de Coquimbo. Al comparar las cifras, respecto del decenio anterior, podemos afirmar que aumentó la cantidad de migrantes. Si bien disminuyó algunos puntos la Región Metropolitana, continúa siendo el principal polo de atracción de los sectores migrantes desde el extremo norte. En esta medición aparece la región de Coquimbo como espacio de atracción de un segmento considerable de la población (ver Tabla 6).

Los resultados del Censo 2012 entregan las características de la población en la nueva Región de Arica y Parinacota creada el 2007. En este caso, consideramos la variable destino de la población que vivía en la zona extremo norte durante el quinquenio 2007-2012. Las cifras poblacionales dadas por el INE en la actualidad se encuentran en proceso de cotejo, para ello se realizará un Censo de carácter abreviado en el 2017.
Tabla 5. Población femenina de 5 años o más: corrientes migratorias observadas, región Tarapacá. 1987-1992. Censo 1992

\begin{tabular}{lc}
\hline $\begin{array}{c}\text { Región residencia habitual } \\
1992\end{array}$ & $\begin{array}{c}\text { Región residencia habitual } \\
\text { en } 1987 \text { (Tarapacá) }\end{array}$ \\
\hline Antofagasta & $18,5 \%(2.780$ hab.) \\
Valparaíso & $14,5 \%(2.175$ hab.) \\
Metropolitana & $36,3 \%(5.442$ hab.) \\
Población total emigrante & 15.011 habitantes \\
\hline
\end{tabular}

Fuente: Instituto Nacional de Estadísticas, Migraciones Internas Regionales 1992-2002.

Tabla 6. Población femenina de 5 años o más: corrientes migratorias observadas, región Tarapacá. 1997-2002. Censo 2002

\begin{tabular}{lc}
\hline $\begin{array}{c}\text { Región residencia habitual } \\
2002\end{array}$ & $\begin{array}{c}\text { Región residencia habitual } \\
\text { en 1997 (Tarapacá) }\end{array}$ \\
\hline Antofagasta & $17,9 \%(3.268$ hab.) \\
Coquimbo & $12,0 \%(2.200$ hab.) \\
Valparaíso & $16,4 \%(2.993$ hab. $)$ \\
Metropolitana & $31,3 \%(5.709$ hab.) \\
Población total emigrante & 18.262 habitantes \\
\hline
\end{tabular}

Fuente: Instituto Nacional de Estadísticas, Migraciones Internas Regionales 1992-2002.

La población masculina, siguiendo la tendencia de las mediciones anteriores, tuvo como destino la Región Metropolitana, seguidas por las de Antofagasta, Tarapacá y Valparaíso. Además, la siguiente Tabla demuestra que el segmento etario con mayores cifras de emigración corresponde entre los 15 y 29 años. Entre las casuales que explican esta movilidad se encuentran las relacionadas con educación, desarrollo laboral y salud, entre otros.

La población femenina tiene como principal destino la Región Metropolitana (ver Tabla 7). Sin embargo, a diferencia del género masculino, el segundo destino corresponde a la zona de Antofagasta. A continuación aparecen las regiones de Tarapacá y Valparaíso. Respecto de la estadística del Censo 2002, en esta ocasión disminuye la cantidad de población femenina migrante a la región de Coquimbo. En cuanto al grupo etario, las cifras son bastante similares entre 15 y 29 años y los 30 y 44 años. El movimiento que implica la migración se produce por diversos factores de orden económico, social o cultural, cuyo propósito es la materialización de expectativas. En ese sentido, estudiamos el desplazamiento geográfico para comprobar la falta de oportunidades en la región durante las últimas décadas (ver Tabla 8). 
Tabla 7. Población masculina de 5 años o más: corrientes migratorias observadas, región Arica y Parinacota. 2007-2012. Censo 2012

\begin{tabular}{lccccccc}
\hline \multicolumn{7}{c}{ Región de residencia habitual abril de 2007 } \\
\hline \multicolumn{1}{c}{ Hombres } & Tarapacá & Antofagasta & Valparaíso & Metropolitana & Población 5 o más & No migrantes & Migrantes Internos \\
\hline 5 a 14 años & 250 & 317 & 159 & 402 & 16.368 & 14.105 & 1.501 \\
15 a 29 años & 398 & 539 & 376 & 1.279 & 27.590 & 22.156 & 4.347 \\
30 a 44 años & 402 & 544 & 262 & 882 & 21.328 & 17.832 & 2.743 \\
45 a 59 años & 205 & 240 & 90 & 314 & 17.070 & 15.608 & 1.095 \\
60 a 74 años & 66 & 69 & 31 & 113 & 10.075 & 9.551 & 378 \\
75 años o más & 20 & 18 & 15 & 23 & 3.367 & 3.196 & 116 \\
Población Total & 1.341 & 1.727 & 933 & 3.013 & 95.798 & 82.448 & 10.180 \\
\hline
\end{tabular}

Fuente: Instituto Nacional de Estadísticas (INE).

Tabla 8. Población femenina de 5 años o más: corrientes migratorias observadas, región Arica y Parinacota. 2007-2012. Censo 2012

\begin{tabular}{lccccccr}
\hline \multicolumn{7}{c}{ Región de residencia habitual abril de 2007 } \\
\hline \multicolumn{1}{c}{ Mujeres } & Tarapacá & Antofagasta & Valparaíso & Metropolitana & Población 5 o más & No migrantes & Migrantes Internos \\
\hline 5 a 14 años & 238 & 309 & 144 & 413 & 16.190 & 13.966 & 1.512 \\
15 a 29 años & 421 & 502 & 216 & 633 & 25.628 & 22.027 & 2.468 \\
30 a 44 años & 367 & 432 & 230 & 673 & 22.636 & 19.519 & 2.293 \\
45 a 59 años & 157 & 198 & 57 & 255 & 19.013 & 17.827 & 858 \\
60 a 74 años & 61 & 68 & 38 & 121 & 11.449 & 10.962 & 360 \\
75 años o más & 29 & 38 & 13 & 75 & 4.792 & 4.519 & 215 \\
Población Total & 1.273 & 1.547 & 698 & 2.170 & 99.708 & 88.720 & 7.706 \\
\hline
\end{tabular}

Fuente: Instituto Nacional de Estadísticas (INE).

Respecto de la caracterización de la persona que migra, por ejemplo en el 2012 el grupo de edad de mayor migración nacional desde la Región de Arica y Parinacota se ubicó entre los 15 y 44 años, es decir, se trataba de población económicamente activa, lo que puede ser apreciado como un efecto negativo para la región de origen, debido a la pérdida de un potencial capital humano, correspondiente a trabajadores en plena edad productiva ${ }^{5}$ (ver Gráfico 13).

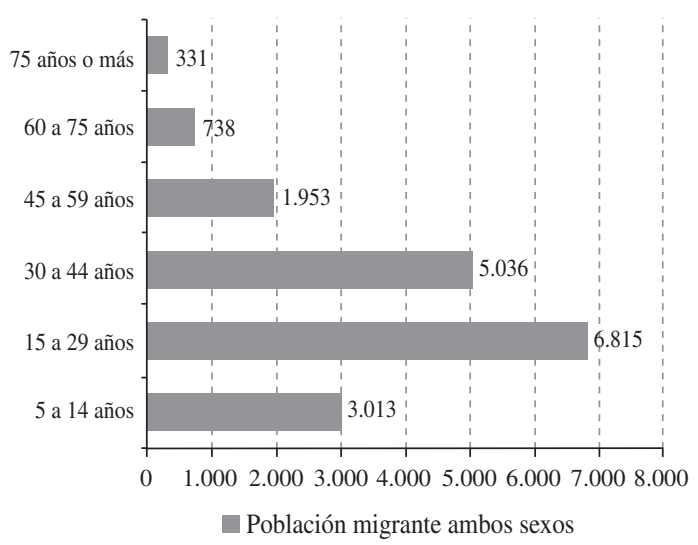

Gráfico 13. Población migrante ambos sexos Región Arica y Parinacota, año 2012.

Fuente: Instituto Nacional de Estadísticas (INE). Censo 2012.

\section{A modo de conclusión}

Desde octubre del 2007, tras la creación de la Región de Arica y Parinacota, se ha venido revirtiendo la tendencia de decrecimiento regional y se han logrado cifras económicas que pueden ser consideradas positivas en términos de desempleo y algunas cifras de crecimiento. Sin embargo, continúa en la población la percepción de una situación de postergación y que desde los gobiernos no ha existido una política dirigida a los espacios fronterizos.

En las páginas de La Estrella, periódico local, es posible leer las opiniones de distintos actores sociales que dan a conocer cuáles son las demandas de la comunidad. Se observa la necesidad de incrementar la inversión en infraestructura pública. Además, se considera un mejoramiento del borde costero, de la construcción de los embalses, de un barrio cívico y otros servicios públicos, de la creación de un nuevo puerto en el sector norte, de la instalación de una planta desaladora. También es una tarea pendiente, para el ámbito político, proyectos vinculados a la educación y empleo que permitan disminuir la emigración del segmento juvenil de la población desde la región hacia la zona central del país. Si bien desde Santiago se señala que para fortalecer la importancia 
estratégica y económica para Chile de esta región, se han promulgado leyes que otorgan incentivos especiales para la misma, ello no se visualiza como una situación permanente y efectivamente transformadora. Según la Cámara de Comercio y la Construcción, la baja sostenida del desempleo en la región se refleja en la falta de mano de obra calificada disponible para los diversos rubros económicos. Según, Luis Maturana, Presidente local de esta institución, los trabajadores disponibles de Arica están prefiriendo la industria minera en otras ciudades antes de la construcción (...) Nosotros venimos advirtiendo esta situación hace mucho tiempo, ya que en la región cuesta mucho encontrar mano de obra calificada para que trabaje en el rubro de la construcción ${ }^{6}$. Por su parte, el presidente de la Cámara de Comercio, Aldo González, ha expresado que tanto los hombres como las mujeres de la ciudad están prefiriendo la industria minera, lo que se aprecia en los precios en el comercio y ventas de inmuebles: Arica se está transformando en una ciudad dormitorio, donde los valores están subiendo considerablemente debido a que, tanto hombres como mujeres, están prefiriendo trabajar en la industria minera que paga mayores salarios ${ }^{7}$.

Mediante diversos estudios se ha recogido la percepción de la población sobre la ciudad. Uno de ellos, realizado por la Universidad Santo Tomás y denominado Percepción de Problemáticas, Condiciones Urbanas y Calidad de Vida en las ciudades de Arica, Iquique, Antofagasta y Copiapó, concluyó que en cuanto a la percepción de calidad de vida de los ariqueños, en 55,25\% (equivalente a 221 personas de los 400 encuestados), la mayoría consideró que era regular, 17,25\% la calificó de mala y muy mala y solo $25,75 \%$ aseguró que era buena. El 1,75\% respondió que era muy buena ${ }^{8}$. Entre las principales problemáticas identificadas se encontraban las de carácter económico y a las expectativas que las personas poseían respecto del crecimiento en la infraestructura de la ciudad. Desde el punto de vista sanitario, se manifestaron preocupaciones sobre la ocurrencia del Sida, situación coincidente con las informaciones de autoridades de Salud sobre el crecimiento sostenido de las tasas de VIH durante los últimos veinte años, afectando principalmente a la población joven.

A modo de comentarios finales podemos señalar que las cifras de la población desde la década de 1990 y las proyecciones hacia el año 2020 en la comunidad de Arica, demuestran que existen cambios en los tres grandes segmentos de la población: una disminución de la natalidad, la migración de los grupos jóvenes y un aumento de los adultos mayores. Las expansiones o restricciones de habitantes establecen varios desafíos en materia de políticas públicas. Justamente en el momento en que el Estado cuenta con mayor cantidad de recursos en su historia, el crecimiento de la población se detiene. En este caso, los desafíos para el Estado en la zona periférica son tres: asegurar una tasa de recambio generacional, aumentar las posibilidades de desarrollo en la región para evitar la fuga de capital humano y la inversión en salud para disminuir los costos de tratar enfermedades en los adultos mayores.

\section{Agradecimientos}

Nuestro agradecimiento al convenio de desempeño UTA-MINEDUC.

\section{Referencias Citadas}

Altamirano, Teófilo

2004 "Transnacionalismo, remesas y economía doméstica".

Becker, Gary, A Treatise on the Family. Harvard, 1994.

Díaz Araya, Alberto; Luis Galdames Rosas; Rodrigo Ruz Zagal y Alejandro Tapia Tosetti.

2012 "El pasado peruano de Arica", Revista Atenea, Universidad de Concepción, Concepción.

Jeldres Gutiérrez, Mario, Demografía y Estadísticas Vitales. Macro Zona Norte, (INE) Las Estadísticas de Chile, Santiago-Chile.

Ortega, José y David S. Rehen, "Nivel de vida, reproducción y salud en América del Sur durante el siglo XX", en: European Review of Latin America and Caribbean Studies, vol. 60, 1996, pp. 31-70.
Ruz Zagal, Rodrigo; Díaz Araya, Alberto y Luis Galdames Rosas 2008 Población Andina de las Provincias de Arica y Tarapacá. El Censo inédito de 1866, Ediciones Universidad de Tarapacá, Arica.

Sánchez, Jesús, El crecimiento de la población mundial. Implicaciones socioeconómicas, ecológicas y éticas, Valencia-España, 2008.

Tapia, Marcela

2012 "Frontera y migración en el norte a partir del análisis de los censos población. Siglos XIX-XXI", Revista de Geografia Norte Grande, 53, pp. 177-198, Santiago.

Thane, Patricia, "The Ageing of modern societies: ¿crisis or opportunity?", en: Historia 396, Pontificia Universidad Católica de Valparaíso, vol. 3 n² 2, pp. 333-349. 
Universidad Santo Tomás, Percepción de Problemáticas, Condiciones Urbanas y Calidad de Vida en las ciudades de Arica, Iquique, Antofagasta y Copiapó, 2012.

\section{Fuentes y documentos:}

Diario La Estrella de Arica, octubre-noviembre del 2012.

Instituto Nacional de Estadísticas (INE)

1999 Panorama Regional. Estadísticas Regionales de Chile 1990-1998, Santiago-Chile.

Instituto Nacional de Estadísticas (INE), Departamento Estadísticas Demográficas y Sociales

1999 Chile y los adultos mayores impacto en la sociedad del 2000, Santiago-Chile.

Instituto Nacional de Estadísticas (INE)

2005 Chile hacia el 2050. Proyecciones de Población, Santiago-Chile.

Instituto Nacional de Estadísticas (INE)

2011 Compendio Estadístico 2011. Estadísticas Demográficas, Santiago-Chile.

Instituto Nacional de Estadísticas (INE)

2012 Encuesta de empleo, período 1986-2012.

Instituto Nacional de Estadísticas (INE),

1992 XVI Censo Nacional de Población y V de Vivienda, Santiago-Chile.

Instituto Nacional de Estadísticas (INE),

2002 XVII Censo Nacional de Población y VI de Vivienda, Santiago-Chile.

Instituto Nacional de Estadísticas (INE),
2012 XVIII Censo Nacional de Población y VII de Vivienda, Santiago-Chile.

Ministerio de Desarrollo Social,

2011 Resultados Regionales Encuesta Casen 2011: Región de Arica y Parinacota, Serie Informes Regionales $n^{\circ} 15$, Santiago-Chile.

Ministerio de Planificación y Cooperación (MIDEPLAN) 2006 Encuesta Casen.

Ministerio de Desarrollo Social,

2011 Resultados Regionales Encuesta Casen 2011: Región de Arica y Parinacota.

Ministerio de Salud (MINSAL)

2006 Encuesta de Calidad de Vida y Salud.

Programa de las Naciones Unidas para el Desarrollo (PNUD) 2000 Desarrollo Humano en las Comunas de Chile, Santiago-Chile.

Programa de las Naciones Unidas para el Desarrollo (PNUD) 1999 Índice de Desarrollo Humano: Chile 1990-1998. Temas de Desarrollo Humano Sustentable, Santiago-Chile.

Secretaría Regional Ministerial de Salud Arica y Parinacota, Unidad de Epidemiología

2008 Informe Epidemiológico VIH/SIDA.

Secretaría Regional Ministerial de Salud Arica y Parinacota, Unidad de Epidemiología

2009 Diagnósticos Regionales con Enfoque DSS Región de Arica y Parinacota.

Subsecretaría de Desarrollo Regional y Administrativo 2012 Observatorio Regional - Arica y Parinacota $N^{o} 1$ y 2.

\section{Notas}

1 Las cifras presentadas en esta investigación corresponden a la incidencia de la pobreza y pobreza extrema. La primera categoría es el indicador utilizado con mayor frecuencia, corresponde al porcentaje de individuos cuyo ingreso sea insuficiente para satisfacer sus necesidades básicas.

2 INE, Encuesta de Empleo, trimestre octubre-diciembre 2008.

3 El coeficiente de Gini mide hasta qué punto la distribución del ingreso entre individuos u hogares dentro de una economía se aleja de una distribución perfectamente equitativa. El coeficiente es un número entre 0 y 1 , donde 0 corresponde a la perfecta igualdad (todos los habitantes tienen los mismos ingresos) y el valor 1 se refiere a la perfecta desigualdad (una persona tiene todos los ingresos y los demás nada).

4 Ministerio de Desarrollo Social, Resultados Regionales Encuesta Casen 2011: Región de Arica y Parinacota.

5 Altamirano, Teófilo (2004). Transnacionalismo, remesas y economía doméstica. Cuadernos Electrónicos de Filosofía del Derecho ${ }^{\circ} 10$, Pontifica Universidad Católica del Perú, pp. 2-31.

6 Diario La Estrella de Arica 02/11/2012.

7 Diario La Estrella de Arica 02/11/2012.

8 Diario La Estrella de Arica 27/10/2012. 
